\title{
EMPLEO DEL PROGRAMA VOLCFLOW PARA SIMULAR FLUJOS PIROCLÁSTICOS DEL VOLCÁN CERRO MACHÍN, TOLIMA, COLOMBIA.
}

\section{USING VOLCFLOW SOFTWARE TO SIMULATE PYROCLASTIC FLOWS FROM THE CERRO MACHÍN VOLCANO, TOLIMA, COLOMBIA.}

\author{
Elsa Adriana Cárdenas Quiroga \\ Geóloga M.Sc, Profesora Asistente, Facultad de Ingeniería, \\ Universidad Militar Nueva Granada, Bogotá, Colombia. \\ elsa.cardenas@unimilitar.edu.co \\ Pahola Porras Rodríguez \\ Ingeniera Mecatrónica, Jóven Investigadora Grupo Geomática Aplicada Facultad de Ingeniería, \\ Universidad Militar Nueva Granada, Bogotá, Colombia. \\ paho756@gmail.com \\ Luz Yolanda Morales Martín Ph.D \\ Profesora Asistente, Facultad de Ingeniería, \\ Universidad Militar Nueva Granada, Bogotá, Colombia. \\ luz.morales@unimilitar.edu.co \\ Fecha de recepción: 13 de diciembre de 2012 \\ Fecha de aceptación: 2 diciembre de 2013
}

\begin{abstract}
RESUMEN
Se presentan los resultados de simular futuros flujos piroclásticos que podría producir el Volcán Cerro Machín, ubicado en la Cordillera Central de Colombia. Esta simulación se realizó con el programa VolcFlow desarrollado por el Laboratorio de Magmas y Volcanes de la Universidad Blaise Pascal, Francia, diseñado especialmente para modelar el comportamiento de este tipo de productos volcánicos, ya que está basado en las características reológicas de esos flujos. Se comparan las áreas que serían potencialmente afectadas por una erupción de ese tipo y los espesores del material que se depositaría con resultados obtenidos de simulaciones realizadas con un Model Builder desarrollado en ARC GIS por el Grupo de Investigación Geomática Aplicada de la Universidad Militar Nueva Granada en Colombia, obteniéndose resultados similares.
\end{abstract}

Palabras clave: Simulación, modelos numéricos, modelos estadísticos, flujos piroclásticos, áreas de influencia. 


\section{ABSTRACT}

Results of simulating pyroclastic flows that could be produced by the Cerro Machín Volcano, located in the Central Cordillera of Colombia, are presented. The simulation was made using the software VolcFlow developed by the Laboratory of Magmas and Volcanoes of the Blaise Pascal University, France, especially designed to model the behavior of this type of volcanic products, as it is based on the rheological characteristics of such flows. Then areas that would be potentially affected and the thickness of the deposited material are compared with the results of the simulations performed with a Model Builder in ARC GIS developed by the research group on applied geomatics of the Military Nueva Granada University, Colombia, with similar results.

\section{Keywords}

Simulation, numerical models, statistical models, pyroclastic flows, areas of influence.

\section{INTRODUCCIÓN}

Los recientes avances tecnológicos en las áreas de la información geográfica han facilitado el modelamiento y representación gráfica de diversos tipos de fenómenos naturales, permitiendo así, responder a las necesidades relacionadas con la planeación y el manejo de los efectos relacionados con la ocurrencia de dichos eventos.

Los sistemas de información geográfica y los programas de simulación permiten el almacenamiento, procesamiento, análisis y representación de la información espacial de áreas que eventualmente podrían resultar afectadas en caso de ocurrir un proceso geológico, como por ejemplo una erupción volcánica, cuyas características hacen difícil modelarlo e interpretarlo para tomar decisiones relacionadas con la planificación y el ordenamiento territorial.
La investigación, atención, prevención y manejo de riesgos asociados a la ocurrencia de eventos naturales involucran el uso de tecnologías computacionales para la simulación de amenazas principalmente de tipo volcánico, y de sistemas de información geográfica para la generación de posibles áreas de afectación.

En Colombia, a raíz de la erupción del Volcán Nevado del Ruiz en 1985, se vio claramente la necesidad de estudiar el comportamiento de los demás volcanes considerados como activos en el país y generar mapas de amenaza y riesgo correspondientes a cada uno de ellos, de acuerdo con la metodología establecida por INGEOMINAS (1995) [1]. A partir de ese momento, se estableció como requisito la utilización de algún sistema de información geográfica (ILWIS), que son las siglas de "Integrated Land and Water Information System". 
Uno de los volcanes objeto de estudio fue el Volcán Cerro Machín (VCM), ubicado en la Cordillera Central de Colombia y considerado como de alta peligrosidad. Dentro del proceso de zonificación de amenazas realizado para dicho volcán se simuló la ocurrencia de diferentes tipos de amenaza a partir de la información geológica, geomorfológica y de parámetros climatológicos e hidrológicos, integrando adicionalmente, información de modelos numéricos digitales VAFTAD (Volcanic Ash Forecast Transport and Dispersión), que contribuyeron a determinar la dispersión de flujos piroclásticos [2].

Posteriormente, en 2003 Obando y Ramos realizaron un trabajo que permitió modelar el comportamiento de flujos piroclásticos para el VCM, empleando el software FLOW 3D [3]. En 2009 Murcia y otros efectuaron simulaciones de flujos piroclásticos para determinar el área afectada por este tipo de amenaza empleando el software TITAN 2D, desarrollado especialmente por la Universidad de Búffalo, para modelar el comportamiento de flujos granulares [4]. En ambos trabajos se generaron los respectivos mapas de amenazas a partir de la simulación de los principales eventos eruptivos registrados.

Con el ánimo de contribuir al conocimiento del comportamiento del VCM, en este trabajo se presentan los resultados de simulaciones empleando el software VolcFlow, desarrollado por la Universidad de Blaise Pascal y creado con el fin de simular flujos piroclásticos. Este software permite combinar diferentes parámetros para proponer los diversos escenarios para una erupción futura, y aporta por tanto, elementos relevantes para la posible toma de decisiones preventivas.

\section{MARCO REFERENCIAL}

\subsection{ERUPCIONES VOLCÁNICAS}

Las erupciones volcánicas son una expresión de la dinámica terrestre que, al igual que otros procesos como los terremotos, ocurren gracias a la interacción entre las placas tectónicas.

La actividad volcánica ocurre principalmente en los límites entre las placas. El tipo de límite y el movimiento relativo de las placas son elementos importantes para determinar el tipo de edificio volcánico que se construirá, el estilo eruptivo y la composición del magma. En límites convergentes, cuando una placa densa se introduce bajo una placa menos densa en el proceso conocido como subducción, ocurre una serie de procesos que favorecen la generación de considerables cantidades de magma y la actividad volcánica que se produce puede ser de tipo explosivo. En los límites divergentes, cuando las placas se alejan, la actividad volcánica típica suele ser menos explosiva y el magma produce nueva corteza oceánica. La actividad volcánica ocurre también en el interior de las placas tectónicas como producto de puntos calientes del manto, conocidos como hot spots, de la que la cadena de islas volcánicas de Hawai es el ejemplo más conocido y estudiado [5]. 


\subsection{PRODUCTOS VOLCÁNICOS}

De acuerdo con la composición química del magma, la actividad volcánica puede ser explosiva o efusiva. En el primer caso, se genera una amplia variedad de productos volcánicos y en el segundo tipo de actividad, los principales productos son derrames de lava de diferentes tipos. El carácter explosivo de la actividad volcánica se debe a que la composición de los magmas es más ácida y son más viscosos, con una mayor resistencia a fluir, razón por la cual se forman usualmente domos que obstruyen el cráter principal y que provocan una acumulación de gases en las porciones superiores de los conductos volcánicos que eventualmente, son destruidos mediante una explosión.

Durante la actividad explosiva las partes solidificadas del magma se rompen y dan lugar a la formación de fragmentos sólidos conocidos como piroclastos, cuyas características dependen principalmente de la composición del magma, del tipo de erupción y de su emplazamiento. Cerca del conducto eruptivo los piroclastos son lanzados como proyectiles balísticos. En otros casos, pueden formarse columnas eruptivas que se elevan varios kilómetros y producen nubes eruptivas compuestas por piroclastos finos y/o ceniza y polvo que se depositan lejos del conducto eruptivo. Estas columnas pueden colapsarse por efecto de la gravedad y dar lugar a los peligrosos flujos piroclásticos.

Los flujos piroclásticos se pueden generar por mecanismos diferentes. Uno de ellos es el mencionado colapso gravitacional de la columna eruptiva, pero también por el colapso de un domo de lava en crecimiento. Los flujos piroclásticos se pueden clasificar de acuerdo con su densidad y composición en flujos de ceniza y pómez, flujos de bloques y cenizas y oleadas piroclásticas.

Los flujos piroclásticos representan la mayor amenaza volcánica porque pueden moverse a gran velocidad sobre las laderas del cono volcánico, arrasando con todo a su paso. Por ello, es importante monitorear continuamente la actividad volcánica, entre otros mecanismos, mediante la interpretación de las señales sísmicas y micro sísmicas. [6]

\subsection{EL PROGRAMA VolcFlow}

Se han desarrollado diferentes tipos de software para modelar los diferentes tipos de amenazas volcánicas. Entre ellos están Hazard, Titan 2D, Vaftad, Flow3D. Sin embargo, sólo VolcFlow fue creado especialmente para simular flujos gravitacionales, por lo que se escogió para el desarrollo de este trabajo. Éste fue desarrollado por Karim Kelfoun del Laboratoire Magmas et Volcans, Université Blaise Pascal, en Clermot Ferrand (Francia), y es el único que permite modelar el comportamiento de flujos y oleadas piroclásticas.

El programa VolcFlow permite simular la trayectoria de flujos densos, flujos diluidos a partir de los flujos densos y la sedimentación de los flujos diluidos, basado en el pro- 
medio vertical de las ecuaciones de conservación de la masa (Ecuación 1) y la cantidad de movimiento (Ecuaciones 2 y 3). Utiliza un sistema de coordenadas relacionado con la topografía del terreno, con $x, y$ paralelos a la superficie topográfica local y $h$ perpendicular. La mayoría de los modelos de flujos granulares se basan en este modelo, el cual es compatible con las evidencias de terreno de la mayoría de las avalanchas de escombros y de flujos piroclásticos densos (ShaIler, 1991; Takarada et al., 1999; van Wyk de Vries et al., 2001, citados por Kelfoun, 2009) [7].

$$
\begin{gathered}
\frac{\partial \mathrm{h}}{\mathrm{dt}}+\frac{\partial}{\partial \mathbf{x}}(\mathrm{hu})+\frac{\partial}{\partial \mathbf{y}}(\mathrm{hv})=0 . \\
\frac{\partial}{\partial t}(\mathrm{hu})+\frac{\partial}{\partial x}\left(h u^{2}\right)+\frac{\partial}{\partial y}(\mathrm{huv})=g h \sin \alpha_{\mathrm{x}}-\frac{1}{2} \text { Kactpass } \frac{\partial}{\partial x}\left(\mathrm{gh}^{2} \cos \alpha\right)+\frac{\mathrm{Tx}}{\rho} \\
\frac{\partial}{\partial t}(\mathrm{hv})+\frac{\partial}{\partial x}(\mathrm{hvu})+\frac{\partial}{\partial y}\left(\mathrm{hv}^{2}\right)=g h \sin \alpha_{\mathrm{y}}-\frac{1}{2} \text { Kactpass } \frac{\partial}{\partial y}\left(\mathrm{gh}^{2} \cos \alpha\right)+\frac{\mathrm{Ty}}{\rho}
\end{gathered}
$$

Las ecuaciones 1 a 3 son resueltas utilizando un método numérico que integra todas las características reológicas con base en el espesor y la velocidad del flujo, el comportamiento de la fricción, y el tipo de flujo viscoso y turbulento, lo que permite que VolcFlow simule la erosión y la sedimentación y logre capturar los choques y las relajaciones (Toro, 2001). Este esquema numérico se puede utilizar en topografías complejas. [7]

\section{METOdOLOGÍA}

Para la ejecución de este trabajo se siguió una metodología que consta de cuatro eta- pas. La primera, es la adquisición de la información a partir de los datos y curvas de nivel proporcionados por el Instituto Geográfico Agustín Codazzi (IGAC). La segunda etapa, fue la generación del Modelo Digital de Elevación (DEM) con el software ARCGIS versión 9.3.1. La tercera, consistió en la revisión de la historia geológica del VCM y de su comportamiento eruptivo, para determinar los parámetros que serían empleados en la cuarta etapa, que fue justamente la correspondiente a la simulación mediante el uso de programa VolcFlow. En la Figura 1, se esquematizan estas cuatro etapas. 


\section{METODOLOGÍA}

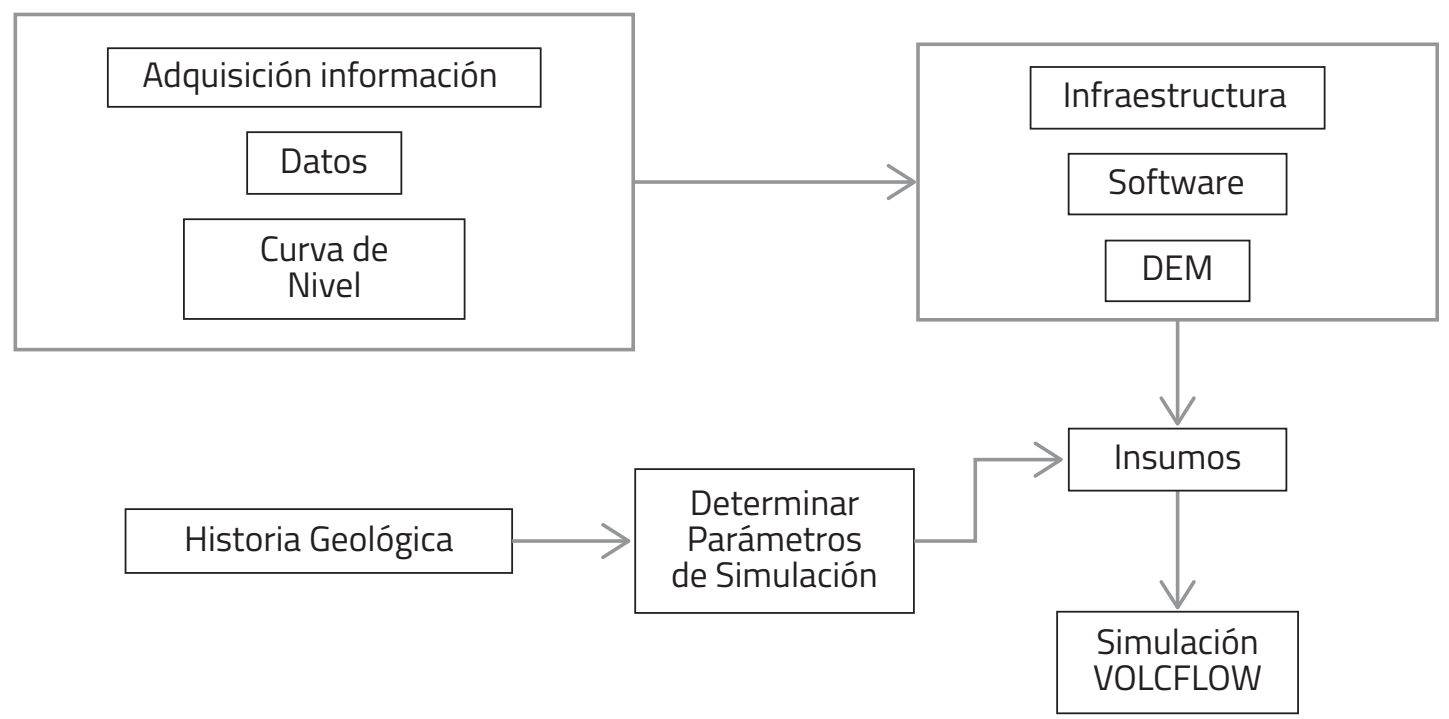

Figura 1. Proceso Metodológico.

La información se obtuvo de las principales bases cartográficas, que serían utilizadas después para la generación del DEM. La principal fuente de información fue la cartografía del IGAC a escala 1:25,000. La segunda fuente de información utilizada fue el ASTER (Advanced Spaceborne Thermal Emission and Reflection Radiometer), que se empleó para completar las áreas sin información de curvas de nivel en las planchas topográficas. Finalmente, para corregir algunas inconsistencias de las curvas generadas por el DEM Aster, se utilizó la cartografía a escala 1:100,000.
Con la información obtenida en la primera etapa se desarrolló el Modelo Digital de Elevación (DEM), utilizando las herramientas del software ARCGIS versión 9.3.1. Desde ArcMap se desplegó la caja de herramientas (Arc Toolbox), se seleccionó la opción Spatial Analyst Tools y finalmente, se aplicó la opción Topo to Raster. En este modelo quedó representada la superficie topográfica del área de influencia requerida para la simulación. La resolución final del DEM, teniendo en cuenta el valor de pixel de salida asignado, fue de $30 \mathrm{~m}$ (Figura 2). 


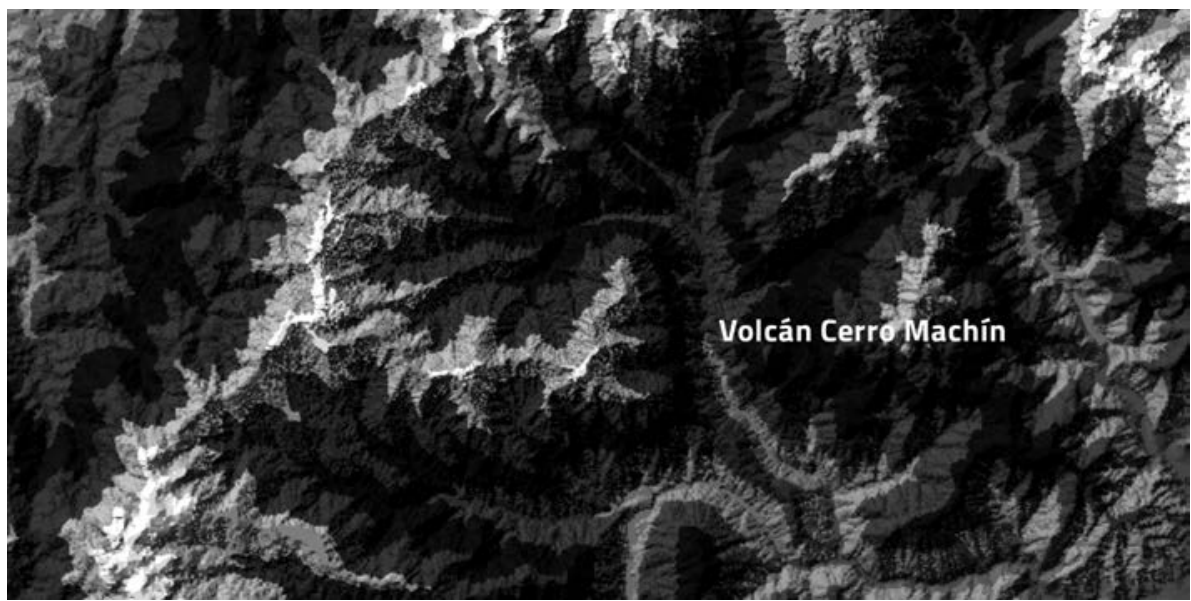

Figura 2. Modelo Digital de Elevación.

Fuente: Elaboración propia.

Durante la tercera etapa, se revisó la información existente relacionada con el historial geológico y eruptivo del VCM, a partir de la cual se escogieron los eventos eruptivos más representativos documentados y caracterizados en trabajos anteriores y se tomaron los valores para las variables que fueron determinadas previamente por Murcia y otros (2010) [4].

Tabla 1. Historial Escenarios Eruptivos Volcán Machín. (Modificada de Murcia y otros, 2010)

\begin{tabular}{|c|c|c|c|c|c|c|c|}
\hline Escenario eruptivo & $\begin{array}{c}10000-5100 \\
\text { años }\end{array}$ & 4800 & 300 años & & & 2500 años & 1200 años \\
\hline Unidades & Unidad Coello & Unidad PO & Unidad Guamo & Unidad P+1 & $\begin{array}{l}\text { Unidad } \\
\text { Espinal }\end{array}$ & $\begin{array}{l}\text { Unidad } \\
\text { Chicoral }\end{array}$ & Unidad P+2 \\
\hline Volumen del depósito & $0,38 \mathrm{~km} 3$ & $0,1 \mathrm{~km} 3$ & $20,18 \mathrm{~km} 3$ & $0,3 \mathrm{~km} 3$ & $0,6 \mathrm{~km} 3$ & $1,35 \mathrm{~km} 3$ & $0,2 \mathrm{~km} 3$ \\
\hline Área del depósito & $25 \mathrm{~km} 2$ & $335 \mathrm{~km} 2$ & $1.000 \mathrm{~km} 2$ & $1.932 \mathrm{~km} 2$ & $294 \mathrm{~km} 2$ & $90 \mathrm{~km} 2$ & $1.210 \mathrm{~km} 2$ \\
\hline $\begin{array}{l}\text { Columna eruptiva } \\
\text { (sobre el cráter) }\end{array}$ & & $26 \mathrm{a} 28 \mathrm{~km}$ & & 36 y $39 \mathrm{~km}$ & & & 33 a 34 km \\
\hline $\begin{array}{l}\text { Distribución columna } \\
\text { (desde el centro) }\end{array}$ & & $26 \mathrm{~km}$ & & $60 \mathrm{~km}$ & & & $45 \mathrm{~km}$ \\
\hline Tiempo estimado de erupción & & $\begin{array}{l}26 \text { y } 51 \\
\text { minutos }\end{array}$ & & 15 y $30 \mathrm{~min}$ & & & 35 a $80 \mathrm{~min}$ \\
\hline Tasa de flujo & & & & $\begin{array}{c}9,3 \times 104 \\
\mathrm{~m} 3 / \mathrm{s}\end{array}$ & & & $\begin{array}{c}7,1 \times 104 \\
\mathrm{~m} 3 / \mathrm{s}\end{array}$ \\
\hline
\end{tabular}


Tabla 2. Características de las unidades eruptivas

\begin{tabular}{|c|c|c|c|c|c|}
\hline Unidades Eruptivas & Edad (años) & Longitud km & Espesor Máximo m & Área km² & Volumen $\mathbf{k m}^{\mathbf{3}}$ \\
\hline Espartillal & 5000 & 8 & 40 & 2,8 & 0,11 \\
\hline PO & 4600 & 7 & 50 & 1,7 & 0,1 \\
\hline P1 & 3600 & 6 & 100 & 18 & 1,8 \\
\hline El Guaico & 2600 & 5 & 50 & 4,5 & 0,3 \\
\hline $\mathrm{P} 2$ & 1200 & 0 & 40 & 2,2 & 0,1 \\
\hline Anillo & 900 & 0 & 1 & 19 & 0,02 \\
\hline
\end{tabular}

Es importante resaltar que la amenaza objeto de estudio son los flujos piroclásticos, típicos de erupciones explosivas, que han sido considerados como una de las amenazas más importantes por su gran peligrosidad.

Ante la imposibilidad para determinar con certeza las características de erupciones futuras, es recomendable utilizar métodos estadísticos para construir un potencial escenario futuro. Para ello, el procedimiento consiste en escoger aleatoriamente parámetros eruptivos dentro de un rango de valores establecidos y efectuar simulaciones relacionadas con tales eventos eruptivos.

Para el caso concreto del VCM, se tomó como base el análisis efectuado por Méndez (2002) [2], en el cual se describen ocho fases características de una erupción potencial. Éstas se determinaron mediante la evaluación de los escenarios eruptivos pasados y por su analogía con el episodio eruptivo del Volcán Monte Pinatubo, Filipinas, en 1991, debido a que se consideró que ambos volcanes presentan ciertas similitudes en su historial eruptivo.
Según Méndez las ocho fases de un escenario eruptivo potencial en el VCM serían las siguientes.

Fase 0: Estado de reposo. En esta fase se espera un cambio progresivo y considerable en el nivel de actividad del volcán.

Fase I: Estado de pre-crisis. Caracterizada por un incremento en la sismicidad, en la tasa de emisión de gases y su composición y en el grado de deformación del terreno.

Fase II: Inicio de crisis. Incremento tanto en la actividad sísmica como fumarólica y emisiones explosivas asociadas a las zonas de debilidad, principalmente en la zona I, y cambios en la composición geoquímica de los manantiales asociados al volcán.

Fase III: Erupciones magmáticas menores. Esta etapa se identifica por un aumento importante en la actividad, caracterizada por el emplazamiento de domos en las zonas de debilidad I y II, aumento de erupciones freáticas, explosiones con generación de flujos piroclásticos menores y deformaciones considerables en el edificio volcánico. 
Fase IV: Explosiones tipo blast. En esta fase ocurrirán erupciones mayores, con columnas eruptivas de aproximadamente $10 \mathrm{~km}$ de altura a partir de las zonas de debilidad I y II, con generación de caídas, oleadas piroclásticas y flujos.

Fase V: Erupción principal o paroxismal. Ocurrencia probable de erupciones plinianas asociadas al colapso o voladura de los domos que ocupan el cráter, con alturas de $25 \mathrm{~km}$, que generarían oleadas y caídas piroclásticas y flujos piroclásticos mayores.

Fase VI: Post-climática o post-paroxismal. Ocurrencia de erupciones explosivas menores.
Fase VII: Final de la erupción. Emplazamiento probable de nuevos domos y presencia de lahares fríos.

De esa secuencia de eventos, se deduce que la actividad volcánica estaría concentrada principalmente en las zonas de debilidad I y II del VCM, cuya ubicación se puede observar en la Figura 3. Estas zonas corresponden a una serie de fracturas de tipo radial que podrían ser los conductos para una nueva actividad eruptiva [2]. Por ello, el origen de los eventos simulados en este trabajo se localizó en esas zonas de debilidad. Para cada una de ellas se realizaron seis simulaciones.

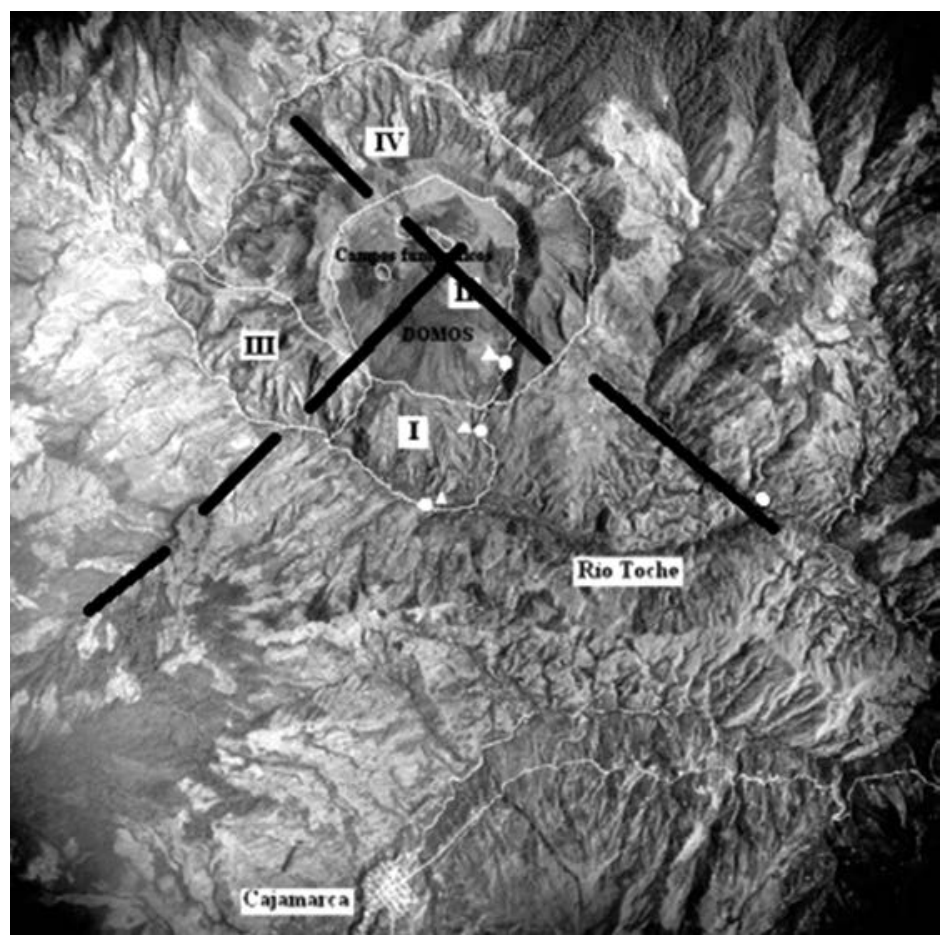

Figura 3. Zonas de debilidad.

Fuente: Méndez et al., 2002 [2]. 


\section{PROCESO DE SIMULACIÓN}

Se desarrollaron alrededor de 40 corridas de simulación en el software VolcFlow, con modificaciones propias en el código para generar los correspondientes mapas de representación de las áreas afectadas por flujos piroclásticos. En esas corridas la densidad fue considerada fija con un valor de $1000 \mathrm{~kg} / \mathrm{m}^{3}$ y se consideró que la sedimentación influye únicamente en el espesor del flujo.
Durante la primera etapa del trabajo se generaron 20 simulaciones combinando distintos rangos de variables, como se observa en la Tabla 3. El área de simulación se definió de acuerdo con la zona de influencia real estimada de afectación por flujos piroclásticos durante una eventual erupción del Volcán Machín, comprendida entre las coordenadas X: 828162, 14742463, Y: 945216, 64204147, y dividida en celdas o pixeles para efectos de la simulación en 2998 columnas: 2998 y 1827 filas.

Tabla 3. Parámetros de las primeras simulaciones realizadas

\begin{tabular}{|c|c|c|c|c|}
\hline Simulación & $\begin{array}{l}\text { Ángulo } \\
\text { Interno }\end{array}$ & Ángulo de Fricción Externo & $\begin{array}{c}\text { Densidad } \\
\left(\mathrm{kg} / \mathrm{m}^{3}\right)\end{array}$ & Viscosidad (Pa.s) \\
\hline 1 & 34 & 5.84 (Espartillal) & 1000 & 0,1 \\
\hline 2 & 34 & 6.74 (Boquerón) & 1000 & 0,1 \\
\hline 3 & 34 & 10.34 (Toche) & 1000 & 0,1 \\
\hline 4 & 34 & 12 (Rosano) & 1000 & 0,1 \\
\hline 5 & 34 & 15 (Murcia) & 1000 & 0,1 \\
\hline 6 & 34 & 5.84 (Espartillal) & 1000 & 1 \\
\hline 7 & 34 & 6.74 (Boquerón) & 1000 & 1 \\
\hline 8 & 34 & 10.34 (Toche) & 1000 & 1 \\
\hline 9 & 34 & 12 (Rosano) & 1000 & 1 \\
\hline 10 & 34 & 15 (Murcia) & 1000 & 1 \\
\hline 11 & 34 & 5.84 (Espartillal) & 1000 & 10 \\
\hline 12 & 34 & 6.74 (Boquerón) & 1000 & 10 \\
\hline 13 & 34 & 10.34 (Toche) & 1000 & 10 \\
\hline 14 & 34 & 12 (Rosano) & 1000 & 10 \\
\hline 15 & 34 & 15 (Murcia) & 1000 & 10 \\
\hline 16 & 34 & 5.84 (Espartillal) & 1000 & 100 \\
\hline 17 & 34 & 6.74 (Boquerón) & 1000 & 100 \\
\hline 18 & 34 & 10.34 (Toche) & 1000 & 100 \\
\hline 19 & 34 & 12 (Rosano) & 1000 & 100 \\
\hline 20 & 34 & 15 (Murcia) & 1000 & 100 \\
\hline
\end{tabular}


Los parámetros tomados en cuenta para la realización de las simulaciones incluyen los siguientes ángulos:

Ángulo de Fricción Interno: En VolcFlow, esta variable define el ángulo de fricción interna entre las partículas del fluido y varía de acuerdo al tipo de flujo que se quiera simular.

Ángulo de Fricción Externa: Esta variable representa el ángulo entre el flujo y la base donde se está desarrollando la erupción.

Para realizar cada simulación se siguió el procedimiento siguiente:
- Lectura del archivo ASCII del DEM, obteniendo el tamaño del gráfico en dos dimensiones a representar. El modelo con el código ASCII se obtuvo por medio de ARCGIS.

- A continuación se ingresaron las características del terreno y del flujo a simular, que varían para cada flujo, tal como se observa en la Tabla 4.

Después de las primeras 20 simulaciones con un solo origen, se decidió realizar otras simulaciones con otro origen localizado en la segunda zona de debilidad.

Tabla 4. Variables ingresadas al software.

\begin{tabular}{|l|l|l|}
\hline \multicolumn{1}{|c|}{ Variable } & \multicolumn{1}{|c|}{ Valor } & Cantidad Representada \\
\hline Delta_int & 0/180*pi & Ángulo interno \\
\hline Delta_bed & $0 / 180^{*}$ pi & Ángulo de lecho \\
\hline Cohesion & 25000 & $\begin{array}{l}\text { Fuerza por unidad de } \\
\text { superficie }\end{array}$ \\
\hline Rho & 2000 & \begin{tabular}{l} 
Densidad \\
\hline Viscosity
\end{tabular} \\
\hline
\end{tabular}

Durante la ejecución de las corridas, se evidenció que existían requerimientos relacionados con los sistemas operativos y la memoria RAM del procesador para garantizar el correcto funcionamiento del software y otras condiciones mínimas para evitar la generación de errores en la simulación. También se presentaron algunos errores que se mencionan a continuación y que merecen ser tomados en cuenta en próximos trabajos que utilicen este software. 
a) Error con el Firewall. La Universidad Militar, donde se desarrolló este trabajo, tiene protección de conexión a servidores externos, por lo que no fue posible realizar las simulaciones en un computador que estuviera en dominio con la red de la Universidad.

b) El software VolcFlow fue licenciado para el Grupo de Investigación Geomática Aplicada alrededor de un mes, después de este tiempo el software indica que la licencia expiró.

c) Los codecs de video del software VolcFlow no son compatibles con los sistemas operativos Windows 7, Windows XP y Linux de 64 bits.

En Windows 7 y Windows XP, si la computadora a utilizar tiene menos de $2 \mathrm{~Gb}$ de memoria RAM, el software genera errores.

Ubuntu de 32 bits: El software corre con una memoria RAM de $4 \mathrm{~Gb}$.

\section{RESULTADOS Y ANÁLISIS}

En la Tabla 5, se presentan como principal resultado las velocidades finales alcanzadas por los flujos piroclásticos simulados a partir de los diferentes puntos de origen, relacionados con las zonas de debilidad identificadas por Méndez (2002) [2]. Para la realización de las pruebas se hicieron algunas variaciones de las variables escogidas para la simulación, tanto en el ángulo de fricción como en el ángulo de lecho, con el fin de identificar la incidencia de este cambio sobre la velocidad máxima final.
Se puede observar que con una viscosidad constante y durante el mismo tiempo de simulación, un incremento en el ángulo de fricción interna y una disminución en el ángulo de lecho, resultan en el aumento de la velocidad máxima final. Este comportamiento es el mismo en cada uno de los orígenes escogidos como punto de inicio de la simulación.

Tabla 5. Resultados de las simulaciones con viscosidad $\mu=0,1 \mathrm{~Pa} / \mathrm{s}$ y $\mathrm{t}=30000$

\begin{tabular}{|c|c|c|c|}
\hline ORIGEN & \begin{tabular}{|c|} 
ÁNGULO DE \\
FRICCIÓN INTERNA \\
33 \\
\end{tabular} & \begin{tabular}{|c|} 
ÁNGULLO DE \\
FRICCIÓN EXTERNA \\
14 \\
\end{tabular} & $\begin{array}{c}\text { VELOCIDAD } \\
\text { MÁXIMA FINAL } \\
2,42 \mathrm{~m} / \mathrm{s}\end{array}$ \\
\hline \multirow{5}{*}{1} & 33 & 15 & $2,40 \mathrm{~m} / \mathrm{s}$ \\
\hline & 33 & 16 & $2,52 \mathrm{~m} / \mathrm{s}$ \\
\hline & 34 & 14 & $2,65 \mathrm{~m} / \mathrm{s}$ \\
\hline & 34 & 15 & $2,59 \mathrm{~m} / \mathrm{s}$ \\
\hline & 34 & 16 & $2,57 \mathrm{~m} / \mathrm{s}$ \\
\hline ORIGEN & \begin{tabular}{|c|} 
ÁNGULO DE \\
FRICCIÓN INTERNA \\
\end{tabular} & \begin{tabular}{|c|} 
ÁNGULO DE \\
FRICCIÓN EXTERNA \\
\end{tabular} & $\begin{array}{c}\text { VELOCIDAD MÁXIMA } \\
\text { FINAL }\end{array}$ \\
\hline \multirow{6}{*}{2} & \begin{tabular}{|l|}
33 \\
\end{tabular} & 14 & $4,49 \mathrm{~m} / \mathrm{s}$ \\
\hline & 33 & 15 & $4,60 \mathrm{~m} / \mathrm{s}$ \\
\hline & 33 & 16 & $4,65 \mathrm{~m} / \mathrm{s}$ \\
\hline & 34 & 14 & $4,93 \mathrm{~m} / \mathrm{s}$ \\
\hline & 34 & 15 & $4,94 \mathrm{~m} / \mathrm{s}$ \\
\hline & 34 & 16 & $4,84 \mathrm{~m} / \mathrm{s}$ \\
\hline ORIGEN & \begin{tabular}{|c|} 
ÁNGULO DE \\
FRICCIÓN INTERNA \\
\end{tabular} & \begin{tabular}{|c|} 
ÁNGULO DE \\
FRICCIÓN EXTERNA \\
\end{tabular} & $\begin{array}{c}\text { VELOCIDAD MÁXIMA } \\
\text { FINAL }\end{array}$ \\
\hline \multirow{6}{*}{3} & 33 & 14 & $1,59 \mathrm{~m} / \mathrm{s}$ \\
\hline & 33 & 15 & $1,72 \mathrm{~m} / \mathrm{s}$ \\
\hline & 33 & 16 & $1,74 \mathrm{~m} / \mathrm{s}$ \\
\hline & 34 & 14 & $1,68 \mathrm{~m} / \mathrm{s}$ \\
\hline & 34 & 15 & $1,83 \mathrm{~m} / \mathrm{s}$ \\
\hline & 34 & 16 & $1,80 \mathrm{~m} / \mathrm{s}$ \\
\hline ORIGEN & \begin{tabular}{|c|} 
ÁNGULO DE \\
FRICCIÓN INTERNA \\
\end{tabular} & \begin{tabular}{|c|} 
ÁNGULO DE \\
FRICCIÓN EXTERNA \\
\end{tabular} & $\begin{array}{c}\text { VELOCIDAD MÁXIMA } \\
\text { FINAL }\end{array}$ \\
\hline \multirow{6}{*}{4} & 33 & 14 & $4,40 \mathrm{~m} / \mathrm{s}$ \\
\hline & 33 & 15 & $4,43 \mathrm{~m} / \mathrm{s}$ \\
\hline & 33 & 16 & $4,39 \mathrm{~m} / \mathrm{s}$ \\
\hline & 34 & 14 & $4,54 \mathrm{~m} / \mathrm{s}$ \\
\hline & 34 & 15 & $4,48 \mathrm{~m} / \mathrm{s}$ \\
\hline & 34 & 16 & $4,40 \mathrm{~m} / \mathrm{s}$ \\
\hline Final & 34 & 16 & $4,40 \mathrm{~m} / \mathrm{s}$ \\
\hline
\end{tabular}


Un segundo resultado lo constituyen los videos de la simulación y las imágenes obtenidas a partir de ellos. (Figuras 4 y 5 ). Las zonas que aparecen con tonos más oscuros representan las zonas donde se acumularían los mayores espesores de material piroclástico.

En las simulaciones los flujos viajan algunos kilómetros desde el origen de la erupción y se depositan alcanzando diferentes espesores de acuerdo con la morfología de la zona. Así, en las áreas donde se encuentran depresiones o valles profundos se acumulan los mayores espesores de material. Los espesores dependen, además, de la naturaleza y densidad de los flujos piroclásticos.

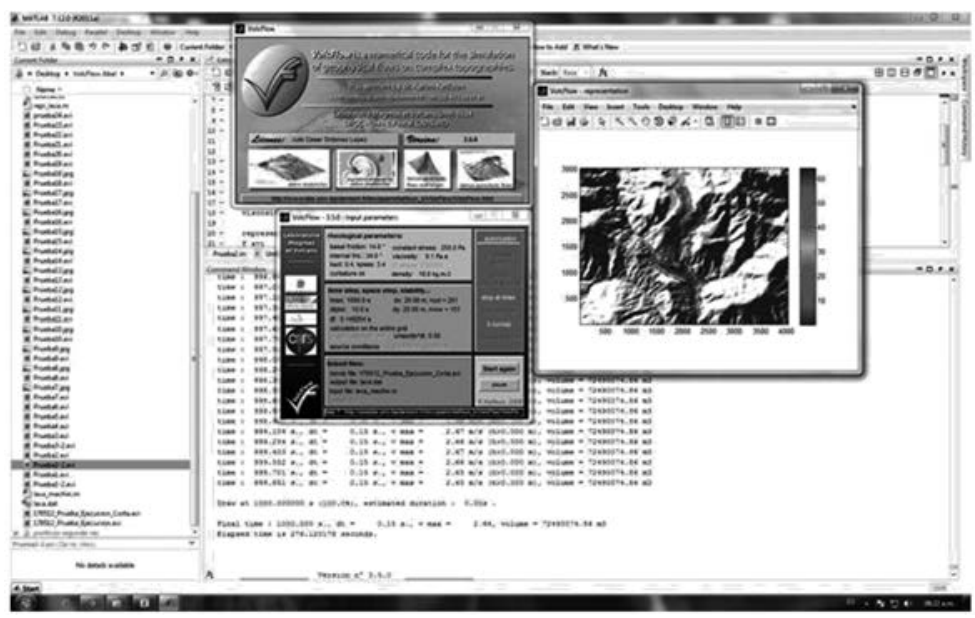

Figura 4. Video de simulación

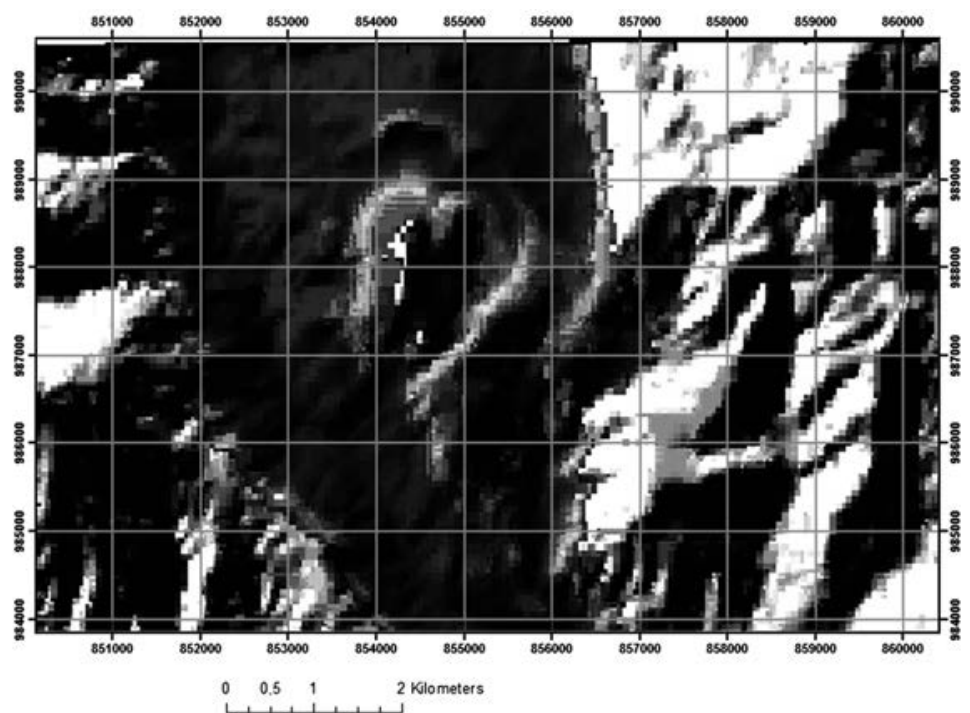

Figura 5. Zonas y espesores alcanzados por los flujos 
Un tercer resultado es la comparación de las áreas de afectación obtenidas a partir de simulaciones previas realizadas a partir del Model Builder (Machín Tool) y las obtenidas ahora con VolcFlow. Es importante aclarar que, a pesar de que el área en la que se corrieron las simulaciones con VolcFlow es más pequeña, debido a que nunca fue posible cargar el DEM del área completa, posiblemente porque los procesadores donde se efectuaron las pruebas no cumplieron con todos los requerimientos, sí existe coincidencia de las zonas donde se acumularían los mayores espesores de material. (Ver Figura 6).

Para efectuar la comparación se escogió la simulación de un evento eruptivo pasado denominado el Espartillal, que se estima fue una erupción de tipo pliniano.

Así, aunque las herramientas computacionales presentan las diferencias que se indican en la Tabla 6, existe una clara coincidencia en el comportamiento que exhibirían los flujos piroclásticos en caso de una futura erupción.

Tabla 6. Comparación entre los métodos empleados para la simulación

\begin{tabular}{|c|c|}
\hline Model Builder (Machín tool) & VolcFlow \\
\hline $\begin{array}{l}\text { 1.Construcción del DEM, y } \\
\text { de la distancia euclidiana } \\
\text { del área a evaluar }\end{array}$ & $\begin{array}{l}\text { 1. Cálculo de los términos fuente: } \\
\text { esfuerzos, condiciones internas del } \\
\text { modelo, cálculo del flujo. }\end{array}$ \\
\hline$\checkmark$ & $\begin{array}{l}\text { 2. Cálculo de los nuevos espesores } \\
\text { y velocidades en el mallado }\end{array}$ \\
\hline $\begin{array}{l}\text { 2.Implementación del } \\
\text { Model Builder construido a } \\
\text { partir del modelo de línea } \\
\text { de Energía en Arcgis } 9.3\end{array}$ & $\begin{array}{l}\text { 3. Cálculo de las velocidades y } \\
\text { formas en la topografía, definición } \\
\text { de algunas condiciones de frontera. }\end{array}$ \\
\hline $\begin{array}{c}\text { 3.Simuáción del Área de } \\
\text { Influencia de Eventos } \\
\text { Históricos }\end{array}$ & $\begin{array}{l}\text { 4.Representación de la simulación } \\
\text { y exportar el video en .avi. }\end{array}$ \\
\hline
\end{tabular}




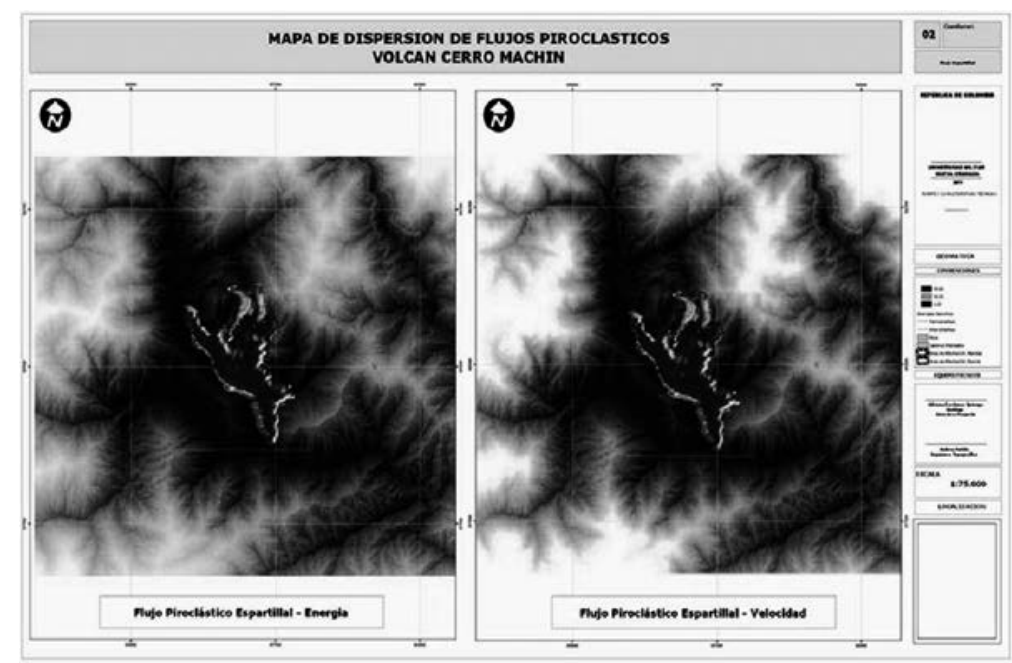

Figura 6. Área de influencia de los flujos piroclásticos y espesores de los depósitos. Fuente: Cárdenas et al. 2012. [9]

\section{CONCLUSIONES}

El software VolcFlow permite simular flujos de baja densidad lo que representa una ventaja en el momento de determinar los espesores reales de los depósitos sobre otros programas de simulación que únicamente, modelan el comportamiento de los flujos densos.

Las herramientas de simulación basadas en el modelo de línea de energía (Modelos numéricos), como el Model Builder (Machín Tool), presentan mayores limitaciones porque simplifican las características topográficas del área de simulación.

Es importante contar con un Modelo Digital de Elevación (DEM) con una alta resolución para garantizar la verosimilitud de los resultados de la simulación, dado que la topografía controla en gran medida tanto las velocidades alcanzadas por los flujos como las zonas donde se acumularían los mayores espesores de los mismos.
Las herramientas computacionales utilizadas en las simulaciones se caracterizan por su versatilidad, permitiendo modificar el código para incluir nuevas variables que favorecerían la obtención de resultados más acordes con la realidad. Incluso, se pueden proponer diferentes escenarios para una erupción futura.

La simulación realizada con VolcFlow permitió definir las zonas donde los flujos piroclásticos alcanzarían las mayores velocidades, coincidentes con las zonas de mayores pendientes y los sitios donde se acumularían los mayores volúmenes de material, que coincidirían con las depresiones topográficas. Estos resultados fueron compatibles con los obtenidos en simulaciones previas con otros programas.

Se puede concluir que en caso de que se presente un evento eruptivo de tipo pliniano, los flujos piroclásticos sobrepasarían barreras topográficas naturales y podrían alcanzar a la población de Cajamarca y otros corregimientos cercanos. 


\section{REFERENCIAS}

[1] Monsalve, M.L.,y Méndez, R.A., 1995. Presentación de la metodología para la elaboración de mapas de amenaza volcánica en Colombia. INGEOMINAS. Disponible en: http://aplicaciones1.ingeominas.gov.co/ Bodega/i_raster/110/02/0000/18130/ documen to/pdf/0101181301101000. pdf

[2] Méndez, R., Cortés, G., y Cepeda, H., 2002. Evaluación de la amenaza volcánica potencial del Cerro Machín (Departamento de Tolima Colombia). INGEOMINAS. Disponible en: http://aplicaciones1.ingeominas.gov.co/Bodega/i_ vector/110/02/0100/02601/documen to/pdf/0101026011101000.pdf

[3] Obando, M., Ramos, G., Cepeda, H., y Portilla, M., 2003. Modelamiento asistido por computador de flujos piroclásticos en el volcán Cerro Machín (Tolima, Colombia). Geología Colombiana 28, pp. 21-37. Disponible en: http:// www.docentes.unal.edu.co/mportillag/ docs/Modelamiento $\% 20$ asistido $\% 20$ po $\quad r \% 20$ computador\%20de\%20flujos\%20pirocl\%E1sticos\%20en\%20el\%20 volc\%E1n\%2 OCerro\%20Mach\%EDn\%

[4] Murcia, H.F., Sheridan, M.F., Macías, J.L., and Cortés, G.P., 2010. TITAN2D simulations of pyroclastic flows at Cerro Machín Volcano, Colombia: Hazard implications.
Journal of South American Earth Sciences 29, pp. 161-170.

[5] Macías, J.L., y Capra, L., 2005. Los Volcanes y sus amenazas. FCE, SEP, CONACyT, México, $159 \mathrm{p}$. Consultado el 10 de diciembre de 2012 en: http://books.google.com.co/ books?id=20ozfR_GP1IC\&printsec=front cover $\& \mathrm{hl}=\mathrm{es} \# \mathrm{v}=$ onepage $\& \mathrm{q} \& \mathrm{f}=$ false

[6] Vyacheslav, M. 2012. Seismic Signals Associated with Pyroclastic Flows, Rockfalls, and Lahars. In: Introduction to Volcanic Seismology (Second Edition), 2012, pp. 261-293.

[7] Kelfoun, K., 2009. Consultoría para la modelización de los flujos piroclásticos del volcán Tungurahua. Proyecto Sistema de Alerta Temprana y Gestión del Riesgo Natural. Consultado el 10 de Diciembre de 2012 en: http://www.igepn.edu.ec/ proyectos/2011_Tungurahua.pdf

[8] Kelfon, K., 2009. VolcFlow. Simulation of volcanic flows. Consultado el 10 de Diciembre de 2012 en: http://wwwobs.univbpclermont.fr/lmv/pperm/kelfoun_k/ VolcFlow/VolcFlow.html

[9] Cárdenas, E.A., y Pulido, A., 2012. Simulación de flujos piroclásticos del Volcán Cerro Machín,Colombia, Mediante la aplicación de herramientas de ARCGIS ${ }^{\circledR}$. En: Ciencia e Ingeniería Neogranadina. Vol 22. PP $63-74$. 